\title{
'Recognising these signs will save lives': Classical Features of a Catastrophic Syndrome
}

\author{
Hamza Hashmi ${ }^{1 *}$, Muhammad Zubair Afzal ${ }^{2}$ \\ ${ }^{1}$ Department of Internal Medicine, Grand Rapids Medical Education Partners, Grand Rapids, Michigan, United States \\ ${ }^{2}$ Department of Internal Medicine, University Of Illinois-Urbana Champaign, Illinois, United States \\ *Corresponding author: hamza.hashmi@grmep.com
}

Received April 24, 2015; Revised May 26, 2015; Accepted June 03, 2015

\begin{abstract}
Ehlers Danlos Syndrome (EDS) type IV is a rare connective tissue disorder that affects skin, visceral and vascular tissue. Diagnosis is often difficult and delayed given absence of hallmark features of a typical EDS. Early recognition of characteristic skin findings may help identify serious and potentially fatal complications. A 19 years old man with a recent spontaneous sigmoid perforation presented with sudden onset of lower abdominal pain. CT abdomen showed left retroperitoneal hematoma. He had worsening abdominal pain, developed hemodynamic instability and suffered a pulseless electrical activity arrest. Labs revealed precipitous drop in hemoglobin and rise in serum lactate level. He was aggressively resuscitated with blood and plasma products. Repeat CT abdomen showed bilateral retroperitoneal hematomas. Abdominal angiogram revealed $23 \mathrm{~mm}$ aneurysmal dilatation in infrarenal aorta. An IR guided intra aortic balloon was inflated to secure hemostasis but before an aortic stent graft could be advanced the friable vessel wall started disintegrating. The patient died within 12 hours of admission. Autopsy revealed typical facies with protruding eyes, thin nose and lips and sunken cheeks; a translucent skin with prominent veins and a transmural dissection of abdominal aorta. Genetic testing revealed classical COL 3A1 missense mutation. Vascular EDS is a diagnostic and therapeutic challenge for both primary care and subspecialty physicians. Diagnosis should be suspected in any young patient with distinctive skin findings and/or spontaneous visceral or arterial rupture. Although no treatment is available knowledge of diagnosis is helpful in management of visceral and vascular complications, pregnancy and genetic counseling.
\end{abstract}

Keywords: Vascular EhlerDanlos Syndrome, pseudoaneurysm, vascular dissection, perforation, acrogeric face

Cite This Article: Hamza Hashmi, and Muhammad Zubair Afzal, "'Recognising these signs will save lives': Classical Features of a Catastrophic Syndrome.” American Journal of Medical Case Reports, vol. 3, no. 7 (2015): 171-174. doi: 10.12691/ajmcr-3-7-2.

\section{Introduction}

Ehlers Danlos Syndrome (EDS) type IV is one of the rare but potentially fatal subtype of Ehler Danlos Syndrome. It is inherited in an autosomal dominant pattern and primarily affects skin, visceral and vascular tissue [1]. It lacks the classical features seen in typical EDS patients and remains undiagnosed until presentation with a life threatening visceral or vascular complication. However these patients have a characteristic facial appearance which may serve as a diagnostic clue. Early recognition of characteristic facial appearance and skin findings may help identify the syndrome and manage potentially fatal complications.

\section{Patient Description}

A 19 years old male presented to the emergency department of our hospital with the chief compliant of sudden onset abdominal pain of one hour duration. His abdominal pain was located in the bilateral lower quadrant and was associated with nausea and lightheadedness. Four months prior to this presentation patient had a spontaneous perforation of sigmoid colon for which he underwent open laparotomy and sigmoid colon resection. He had an uncomplicated postoperative course except for delayed wound healing. He was otherwise a healthy college student with no significant medical, surgical, social and family history.

Initial physical examination revealed stable vital signs but moderate tenderness to palpation in bilateral lower abdominal quadrants. Moreover, he was found to have noticeable features of protruding eyes, thin lips and nose and prominent veins on the chest. Laboratory results revealed hemoglobin of $12 \mathrm{~g} / \mathrm{dl}$ but otherwise normal complete blood count, basic metabolic panel and coagulation profile. Initial CT abdomen showed left sided retroperitoneal hematoma. Imaging results were reviewed by Interventional Radiology and patient was admitted to the Internal Medicine service with recommendations for conservative management and close monitoring of hemodynamic status. Meanwhile, his abdominal pain was controlled by IV pain medication.

About three hour after admission patient suffered sudden pulseless electrical activity arrest. He received two 
minutes of cardiopulmonary resuscitation before return of spontaneous circulation. He was found be markedly hypotensive (BP 70/30) and tachycardic(HR 110 beats / min). Repeat complete blood count revealed precipitous drop in hemoglobin from $12 \mathrm{~g} / \mathrm{dl}$ at time of admission to 6 g/dl post PEA arrest. Repeat CT abdomen showed bilateral retroperitoneal hematomas.

\begin{tabular}{|l|c|c|}
\hline Laboratory Study & Time of Admission & 3 hour after admission \\
\hline Hemoglobin & 12 & 6 \\
\hline Hematocrit & 35 & 19 \\
\hline Platelets & 200 & 79 \\
\hline Potassium & 4.5 & 5.5 \\
\hline Bicarbonate & 25 & 12 \\
\hline pH & & 7.1 \\
\hline Lactate & & 9 \\
\hline Fibrinogen & & $<50$ \\
\hline INR & 1.0 & 1.6 \\
\hline
\end{tabular}

Table showing significant labs at time of admission and 3 hours later
Patient was aggressively resuscitated with blood and plasma products. Emergent abdominal angiogram was performed and revealed a $23 \mathrm{~mm}$ aneurysmal dilatation in infrarenal portion of abdominal aorta. Diffuse extravasation of contrast was seen. An IR guided intra aortic balloon was inflated to secure hemostasis but before an aortic stent graft could be advanced to the site of the pseudoaneurysmthe tissue paper thin friable vessel wall started disintegrating. As there was a clear and distinguishable dissection of aorta, it was considered non salvageable at this point. The patient was made comfort care and passed away within 12 hours of admission. Autopsy requested by the family members revealed atypical facial appearance with protruding eyes, thin nose and lips and sunken cheeks; a translucent skin with prominent veins on the chest and transmural dissection of infrarenal portion of abdominal aorta. Genetic testing done on vascular tissue revealed classical Collagen 3A1 missense mutation classically seen in vascular EhlerDanlos Syndrome patients.



A. CT abdomen showing unilateral left sided retroperitoneal hematoma. No obvious pseudoaneurysm is seen



B. CT abdomen showing worsening bilateral retroperitoneal hematomas 3 hours later 


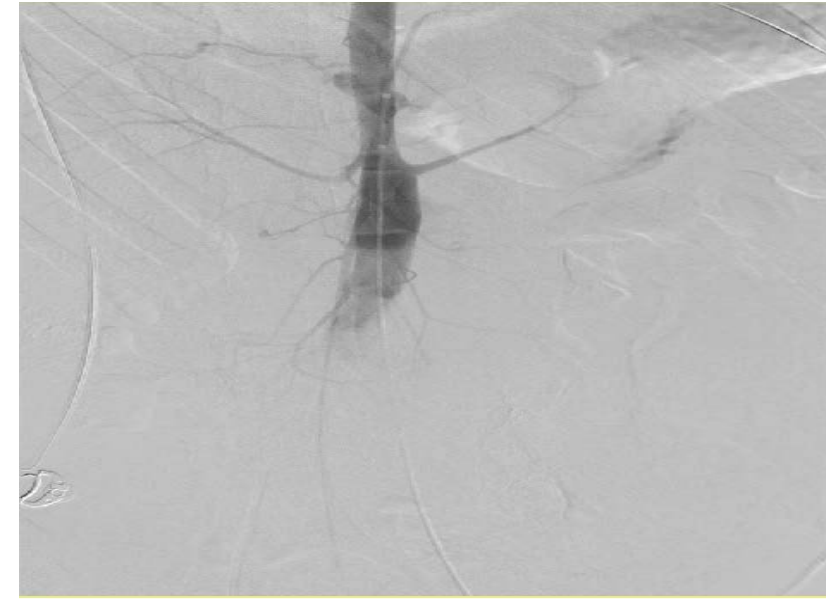

C- Pseudoaneurysm in the infra renal portion of abdominal aorta



D. Psuedoaneurysm with extravasation of dye in the infrarenal portion of abdominal aorta

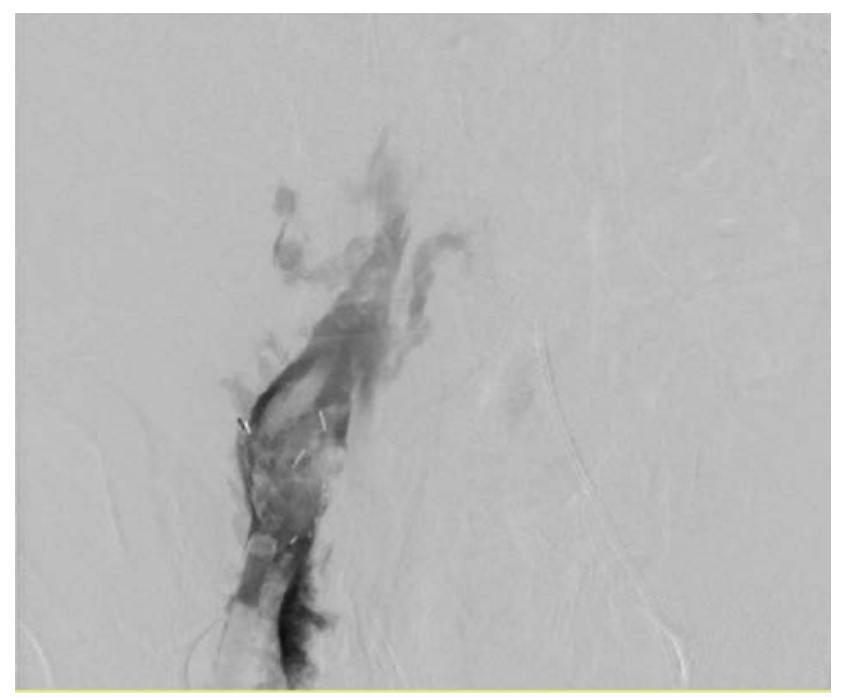

E. Dissection of Pseudoaneurysm (previously shown) and infra renal portion of abdominal aorta with diffuse extravasation of dye upon advancing the aortic stent graft

\section{Discussion}

Ehlers-Danlos Syndrome is a genetic disorder caused by mutations in the genes regulating collagen synthesis resulting in skin, joints, visceral and vascular complications. It is characterized by cardinal features of hyperextensible skin, hypermobile joints and tissue fragility. It has at least six known subtypes but EDS type IV also known as vascular EDS is considered to be of significant clinical relevance because of its potentially life threatening complications [1]. It is inherited in an autosomal dominant pattern and is characterized by missense mutation in COL3A1 gene encoding for procollagen that primarily affects vascular and visceral tissue.Prevalence of all EDS varies between 1/10,000 and $1 / 25,000$. Type IV EDS represents 5 to $10 \%$ of all cases of EDS [2]. Given the absence of distinct features of hyperextensible skin and hypermobile joints commonly seen in other types of EDS, diagnosis is often difficult in a particular case of vascular EDS and is often made after a life threatening complication or at autopsy. Nevertheless at least $30 \%$ of the patients with vascular EDS have a characteristic facial appearance known as 'acrogeric face' which is characterized by thin delicate pinched nose, hollow cheeks, protruding eyes and lobeless ears [3]. These features appear early in life and give a striking appearance to these patients. Other hallmark features of the syndrome include easy bruising, translucent skin with visible veins, rupture of visceral and/or vascular structures and a positive family history. Small joints of fingers and toes may show joint laxity but large joints are usually spared in vascular EDS. Diagnosis is based on a major and minor criteria as mentioned in the table. Diagnosis can be confirmedeither by identification of mutation in the gene for type 3 Pro-collagen (COL3A1) typically seen in arterial wall, skin, uterine and intestinal wall or by demonstrating synthesis of abnormal collagen by cultured dermal fibroblasts.

Complications commonly involve vascular and visceral structures. Average age of first occurrence is 23 years. About $25 \%$ of patients developat least one complications before 20 years of age and more than $80 \%$ of the patients have developed a complication by the time they are 40 years old. Average age of survival is 48 years. Arterial Rupture is responsible for most of the deaths (78.5\%) followed by uterine rupture (10\%) and gastrointestinal perforation (7.5\%). Vascular complications include dissecting aneurysm, major arterial rupture and arterialvenous fistulas [4]. Gastrointestinal complications most commonly involve spontaneous rupture of the sigmoid colon. The episode is fatal in $2 \%$ of the cases and also has a high rate of recurrence. Inguinal, umbilical and hiatal hernia are also commonly seen. Obstetrical complications include uterine rupture during labor and vascular rupture in postpartum period.

Vascular or visceral complications require immediate hospitalization and observation in intensive care unit. Bowel perforation is often managed with a conservative approach. Endovascular treatment is recommended for selective embolization of bleeding vessels and occlusion of carotid cavernous fistula. Invasive surgery is only used as a last resort [5]. When vascular bypass is to be performed venous bypass grafts are preferred [6]. Limited data is available for management of obstetrical complications. Patients are advised to undergo scheduled Cesarean section after 32 weeks and avoid spontaneous vaginal delivery. Given increased risk of uterine perforation, Intra uterine devices should be avoided for contraception purposes [7]. According to the recommendations of European Society of Cardiology 
Type IV EhlerDanlos Syndrome is a contraindication for pregnancy. Several prophylactic measures are recommended to avoid potentially fatal complications from apparently benign causes. Rectal temperature probes and colonoscopies should be avoided. Arterial puncture and central venous line insertion procedures have a higher risk of bleeding complications. Use of beta blocker celiprolol is associated with three fold decrease in arterial rupture in vascular EDS patients [5]. Once one family member is diagnosed with vascular EhlerDanlos Syndrome, all family members need comprehensive genetic testing as well reproductive counseling and long term follow up with clinical geneticist to avoid complications.

\section{Conclusion}

Vascular EDS is a diagnostic and therapeutic challenge for both primary care and subspecialty physicians. Diagnosis is often delayed even when the presentation is typical. Primary care physicians need to be familiar with vascular EDS features as facial appearance and skin findings might be the first signs of the disease. They appear early in life and often before any life threatening complications have occurred. Diagnosis should be suspected in any young patient ( $<40$ years of age) with distinctive skin findings and/or spontaneous visceral or arterial rupture. Recognition of the facial features and skin changes can guide genetic testing and counseling. Although no definitive treatment options are available for EDS type IV, early diagnosis may predict visceral and vascular involvement and affect management of catastrophic complications. Knowledge of the diagnosis is particularly important for counseling females of child bearing age regarding pregnancy and genetic testing of other family members.

\section{References}

[1] Classical features of vascular EhlerDanlos Syndrome [Ferri's clinical advisor 2015.

[2] [Orphanet J Rare Dis. 2007 Jul 19; 2: 32. Ehlers-Danlos syndrome type IV].

[3] [The clinical and genetic features of EhlerDanlos SyndromeVascular Type; NEJM 2000 Mar 9; 342 (10): 673-80].

[4] [Zelochi. M. et al. AM. J Roentgenol. 2007; 189:712-719].

[5] [CurrOpinCardiol. 2011 Nov; 26(6):494-501.

[6] [The spectrum, management and clinical outcome of EhlersDanlos syndrome type IV: a 30-year experience. OderichGS(1), Panneton JM, Bower TC, Lindor NM, Cherry KJ, Noel AA, Kalra M, Sullivan T, Gloviczki P. (1) Division of Vascular Surgery and Department of Medical Genetics, Mayo Clinic, USA.].

[7] A maternal and perinatal mortality in pregnancy complicated by the kyphoscolioticform of Ehlers-Danlos syndrome. [Obstet Gynecol. 2009 Feb; 113(2 Pt 2):515-8. 